\title{
Evolution of Aerosol Particles in the Rainfall Process via Method of Moments
}

\author{
Fangyang Yuan and Fujun Gan \\ School of Aeronautics and Astronautics, Zhejiang University, Hangzhou 310027, China \\ Correspondence should be addressed to Fangyang Yuan; fyyuan.vip@gmail.com
}

Received 20 July 2013; Accepted 13 August 2013

Academic Editor: Jianzhong Lin

Copyright (C) 2013 F. Yuan and F. Gan. This is an open access article distributed under the Creative Commons Attribution License, which permits unrestricted use, distribution, and reproduction in any medium, provided the original work is properly cited.

The method of moments is employed to predict the evolution of aerosol particles in the rainfall process. To describe the dynamic properties of particle size distribution, the population balance equation is converted to moment equations by the method of moments and the converted equations are solved numerically. The variations of particle number concentration, geometric mean diameter, and geometric standard deviation are given in the cases that the Brownian diffusion and inertial impaction of particles dominate, respectively. The effects of raindrop size distribution on particle size distribution are analyzed in nine cases. The results show that the particle number concentration decreases as time goes by, and particles dominated by Brownian diffusion are removed more significantly. The particle number concentration decreases much more rapidly when particle geometric mean diameter is smaller, and the particle size distribution tends to be monodisperse. For the same water content, the raindrops with small geometric mean diameters can remove particles with much higher efficiency than those with large geometric mean diameters. Particles in the "Greenfield gap" are relatively difficult to scavenge, and a new method is needed to remove it from the air.

\section{Introduction}

Our surroundings are filled with aerosol particles which not only affect the environment such as the air visibility, weather, and climate, but also cause respiratory diseases. Available researches show that the respiratory diseases are not only related to the particle mass concentration but also to the particle size and number concentration [1]. Therefore, it is necessary to remove aerosols from air. In nature, precipitation is one of the most effective approaches to remove aerosols in air, in which raindrops collide with aerosols and then collect them. The removing process is affected by external factors including aerosol size distribution [2], raindrop size distribution (RSD) [3], rainfall intensity [4], and physical and chemical properties [5] and internal factors including collision mechanisms between raindrops and aerosols and condensation/evaporation of raindrops and aerosols [6]. The mechanisms involved in the above processes include particle Brownian diffusion, direction interception, inertial impaction, thermo- and diffusiophoresis forces, and electrical forces [2,7]. Although these forces are coupled, one or more of them may dominate for various regions of particle size, drop size, particle density and hydrodynamic temperature, and diffusion fields.

In the rainfall process, the most interesting thing is how the particle size distribution (PSD) changes as time progresses and how PSD is affected by different RSD. In order to answer the questions, the population balance equation (PBE) for particles is introduced [8]. The kernel of $\mathrm{PBE}$ is how to express the scavenging coefficient which represents the removing rate of aerosols by raindrops and is a function of collision efficiency of raindrops, terminal velocity of raindrops, and PSD [4]. In the pioneering work, Slinn [9] obtained a semiempirical formula of collision efficiency according to the Navier-Stokes equation using the dimensionless analysis coupled with the experimental data, which has been widely used when referring to the belowcloud scavenging process. Chate and Kamra [6] showed that the collision efficiency of water drops increases with the increasing impaction parameter. Chate et al. [5] evaluated the scavenging coefficient for aerosols of diameters in the range of $0.02-10 \mu \mathrm{m}$ with various densities in accordance with their chemical compositions for heavy rain regime. They found that the inertial impaction mechanism is the dominant 
one in removing particles of all sizes for the heavy rain regime, and the scavenging coefficient is highly dependent on relative humidity for hygroscopic particles. Mircea and Stefan [4] obtained an exponential expression of the scavenging coefficient as a function of rainfall intensity and collision efficiency between raindrops and aerosol particles. In their later work, Mircea et al. [8] got the linear relations between the scavenging coefficient and the rainfall intensity via numerical analysis. Andronache $[10,11]$ concluded that the below-cloud scavenging (BCS) coefficients of aerosols by rainfall depend mainly on the aerosol size distribution parameters and on rainfall intensity, decreasing significantly with aerosol diameter, increasing with rainfall rate, and average raindrop and aerosol electric charge. Later, Andronache et al. [12] developed a more complicated model to predict the scavenging coefficient, finding it sensitive to the choice of representation of mixing processes, raindrop size distribution, phoretic effects in aerosol-raindrop collisions, and cloud droplet activation.

The research mentioned above was focused on getting the relation between the scavenging coefficient and the external factors. Jung et al. [13] expressed the collision efficiency as polynomial expression of particle diameter and applied the method of moments (MOM) to get analytical solutions of PBE. In their following work [3], they employed the collision efficiency proposed by Slinn [9] and applied the MOM to study the evolution of PSD when the RSD obeys the MarshallPalmer (MP) and Krigian-Mazin (KM) distributions, and they got relatively good results. Besides, Bae et al. [14] developed a good analytical expression for scavenging coefficient. The value of scavenging coefficient can be calculated when the initial three key parameters of PSD and rainfall intensity are given. While in the scavenging process, these parameters evolve as time goes by and the initially calculated scavenging coefficient will be inaccurate due to the evolution of the PSD, even if the rainfall intensity remains the same. In fact, the three parameters of PSD and the scavenging coefficient couple together. Thus, we suggest that more attention should be paid to the dynamic evolution of PSD before getting the value of the scavenging coefficient.

The method of moments has been extensively used to deal with the PBE when referring to physical/chemical changes of particles, including aggregation/breakage [15], condensation/evaporation [16], coagulation $[17,18]$, deposition/removal [19-21], and chemical reaction [22, 23]. The periodic moment method (PMM), proposed by Pratsinis [24], can be used to solve the evolution of PSD for simultaneous nucleation, condensation, and coagulation in the entire particle size spectrum through approximating the size distribution by a unimodal log-normal function.

In the present study, the PMM is adopted to deal with the PBE when referring to the wet scavenging process, and the scavenging coefficient is expressed as a polynomial function of aerosol diameter, raindrop diameter, and raindrop velocity. The evolutions of PSD are simulated numerically and the effects of RSD on PSD are studied. The variations of particle number concentration, geometric mean diameter, and geometric standard deviation are given when Brownian diffusion and inertial impaction of particles dominate, respectively.

\section{Theory}

2.1. Basic Theory of Wet Removal. The governing equation describing the time-dependent removal of particles in air by collision with raindrops [8] is as follows:

$$
-\frac{\partial n\left(d_{p}, t\right)}{\partial t}=\Lambda\left(d_{p}\right) \cdot n\left(d_{p}, t\right),
$$

where $n\left(d_{p}, t\right)$ is the particle size distribution in air and $\Lambda\left(d_{p}\right)$ is the scavenging coefficient representing the rate of scavenged particles by raindrop; consider

$$
\Lambda\left(d_{p}\right)=\int_{0}^{\infty} K\left(d_{p}, D_{d}\right) E\left(d_{p}, D_{d}\right) d D_{d}
$$

where $K\left(d_{p}, D_{d}\right)$ is the collision kernel (or collection kernel) which describes the probability of collisions between particles with diameter $d_{p}$ and raindrops with diameter $D_{d}[3,13]$; consider

$$
K\left(d_{p}, D_{d}\right)=\frac{\pi D_{d}^{2}}{4} U\left(D_{d}\right) n\left(D_{d}\right)
$$

where $E\left(d_{p}, D_{d}\right)$ is the collision efficiency which will be discussed below. $U\left(D_{d}\right)$ is the velocity of a falling raindrop with diameter $D_{d}$ and can be expressed as follows:

$$
\begin{gathered}
U\left(D_{d}\right)=30.75 D_{d}^{2} \times 10^{6}, \quad D_{d}<100 \mu \mathrm{m}, \\
U\left(D_{d}\right)=38 D_{d}^{2} \times 10^{3}, \quad 100 \mu \mathrm{m}<D_{d}<1000 \mu \mathrm{m}, \\
U\left(D_{d}\right)=133.046 D_{d}^{0.5}, \quad D_{d}>1000 \mu \mathrm{m} .
\end{gathered}
$$

The collision efficiency, $E\left(d_{p}, D_{d}\right)$, represents the ratio of the actual frequency of collisions to the theoretical frequency and is affected by turbulent diffusion, Brownian diffusion, van der Waals force, thermophoresis, electrostatic adsorption, and so on. Mircea et al. $[4,8]$ suggested the collision efficiency to be a constant or a function of rainfall intensity (including linear and power law functions). Jung et al. [13] got a set of analytical solutions for polydispersed particles by a wet removal process for submicrometer particles. Slinn [9] obtained the following semiempirical formula according to the Navier-Stokes equation using the dimensionless analysis coupled with the experimental data:

$$
\begin{aligned}
E\left(d_{p}, D_{d}\right)= & \frac{1}{\operatorname{Re} \cdot S \mathrm{Sc}}\left[1+0.4 \mathrm{Re}^{1 / 2} \mathrm{Sc}^{1 / 3}+0.16 \mathrm{Re}^{1 / 2} \mathrm{Sc}^{1 / 2}\right] \\
& +4 \frac{d_{p}}{D_{d}}\left[\frac{\mu_{a}}{\mu_{w}}+\left(1+\mathrm{Re}^{1 / 2}\right) \frac{d_{p}}{D_{d}}\right] \\
& +\left(\frac{\mathrm{St}-S^{*}}{\mathrm{St}-S^{*}+2 / 3}\right)^{3 / 2},
\end{aligned}
$$

where $\operatorname{Re}=D_{d} U\left(D_{d}\right) \rho_{a} /\left(2 \mu_{a}\right)$ is the Reynolds number based on the raindrop diameter, $\mathrm{Sc}=\mu_{a} /\left(\rho_{a} D_{\text {diff }}\right)$ is the Schmidt number of particles with the diffusion coefficient $D_{\text {diff }}=$ $k_{b} T C_{c} /\left(3 \pi \mu_{a} d_{p}\right), \mathrm{St}=2 \tau U\left(D_{d}\right) / D_{d}$ is the Stokes number of 
particles with relaxation time $\tau=\rho_{p} d_{p}^{2} /\left(18 \mu_{a}\right)$, and $S^{*}=$ $[1.2+\ln (1+\mathrm{Re}) / 12] /[1+\ln (1+\mathrm{Re})]$ is a dimensionless parameter. Here, $\rho_{a}$ and $\mu_{a}$ are the density and the viscosity of air, respectively, $\mu_{w}$ is the viscosity of a water drop, $k_{b}$ is Boltzmann's constant, $T$ is the absolute temperature of air, and $C_{c}$ is the Cunningham slip correction factor and can be approximated as follows [25]:

$C_{c}=1+2.493 \frac{\lambda}{d_{p}}+0.84 \frac{\lambda}{d_{p}} \exp \left(-0.435 \frac{d_{p}}{\lambda}\right) \cong 1+3.34 \frac{\lambda}{d_{p}}$,

where $\lambda$ is the molecular mean free path.

The terms on the right-hand side (RHS) of (5) represent the effects of Brownian diffusion, interception, and inertial impaction, respectively.

2.2. Application of the Method of Moments. According to (2)-(3), the scavenging coefficient, $\Lambda\left(d_{p}\right)$, is related to the collision kernel between particles and raindrops, collision efficiency $E\left(d_{p}, D_{d}\right)$, and RSD $n\left(D_{d}\right)$. The RSD based on particle diameter can be described with the log-normal distribution [26], and the particle size distribution can also be approximated by the log-normal distribution [27] as follows:

$$
\begin{aligned}
& n\left(D_{d}\right)=\frac{N_{d}}{\sqrt{2 \pi} \ln \sigma_{d g}} \exp \left[-\frac{\ln ^{2}\left(D_{d} / D_{d g}\right)}{2 \ln ^{2}\left(\sigma_{d g}\right)}\right] \frac{1}{D_{d}}, \\
& n\left(d_{p}\right)=\frac{N_{p}}{\sqrt{2 \pi} \ln \sigma_{p g}} \exp \left[-\frac{\ln ^{2}\left(d_{p} / d_{p g}\right)}{2 \ln ^{2}\left(\sigma_{p g}\right)}\right] \frac{1}{d_{p}},
\end{aligned}
$$

where $N_{d}, D_{d g}$, and $\sigma_{d g}$ are the number concentration, geometric mean diameter, and geometric standard deviation of raindrop, respectively. Definitions of the $k$ th moment of raindrops and particles are $\xi_{k}$ and $m_{k}$, respectively [24]; consider

$$
\begin{aligned}
& \xi_{k}=\int_{0}^{\infty} D_{d}^{k} n\left(D_{d}\right) d D_{d}=\xi_{0} D_{d g}^{k} \exp \left[\frac{k^{2}}{2} \ln ^{2} \sigma_{d g}\right], \\
& m_{k}=\int_{0}^{\infty} d_{p}^{k} n\left(d_{p}\right) d d_{p}=m_{0} d_{p g}^{k} \exp \left[\frac{k^{2}}{2} \ln ^{2} \sigma_{d p}\right] .
\end{aligned}
$$

The last term on RHS of (5) is difficult to deal with when applying the moment method to (1). Thus, the approximate expression proposed by Jung et al. [3] is adopted as follows:

$$
\left(\frac{\mathrm{St}-\mathrm{S}^{*}}{\mathrm{St}-\mathrm{S}^{*}+2 / 3}\right)^{3 / 2} \cong 1-0.9 \mathrm{St}^{-0.5}
$$

The final expression of scavenging coefficient and governing moment equation are as follows $[3,14,28]$ :

$$
\begin{aligned}
\Lambda\left(d_{p}\right)= & \gamma_{1} \xi_{1}\left(d_{p}^{-1}+A d_{p}^{-2}\right)+\gamma_{2} \xi_{7 / 4}\left(d_{p}^{-2 / 3}+2 A d_{p}^{-5 / 3} / 3\right) \\
& +\gamma_{3} \xi_{7 / 4}\left(d_{p}^{-1 / 2}+A d_{p}^{-3 / 2} / 2\right)+\gamma_{4} \xi_{3 / 2} d_{p}+\gamma_{5} \xi_{1 / 2} d_{p}^{2} \\
& +\gamma_{6} \xi_{5 / 4} d_{p}^{2}+\gamma_{7} \xi_{5 / 2}+\gamma_{8} \xi_{11 / 4} d_{p}^{-1}
\end{aligned}
$$

$$
\begin{aligned}
-\frac{\partial m_{k}}{\partial t}= & \gamma_{1} \xi_{1}\left(m_{k-1}+A m_{k-2}\right)+\gamma_{2} \xi_{7 / 4} \\
& \times\left[m_{k-2 / 3}+2 A m_{k-5 / 3} / 3\right]+\gamma_{3} \xi_{7 / 4} \\
& \times\left[m_{k-1 / 2}+A m_{k-3 / 2} / 2\right]+\gamma_{4} \xi_{3 / 2} m_{k+1} \\
& +\gamma_{5} \xi_{1 / 2} m_{k+2}+\gamma_{6} \xi_{5 / 4} m_{k+2} \\
& +\gamma_{7} \xi_{5 / 2} m_{k}+\gamma_{8} \xi_{11 / 4} m_{k-1},
\end{aligned}
$$

where

$$
\begin{aligned}
& \gamma_{1}=\frac{k_{b} T}{6 \mu_{a}}, \quad \gamma_{2}=\left(\frac{0.4 \times 130 \pi}{4}\right)\left(\frac{2 \mu_{a}}{130 \rho_{a}}\right)^{1 / 2}\left(\frac{k_{b} \rho_{a} T}{3 \pi \mu_{a}^{2}}\right)^{2 / 3}, \\
& \gamma_{3}=\left(\frac{0.16 \times 130 \pi}{4}\right)\left(\frac{2 k_{b} T}{3 \times 130 \pi \mu_{a}}\right)^{1 / 2}, \quad \gamma_{4}=\frac{130 \pi \mu_{a}}{\mu_{w}}, \\
& \gamma_{5}=130 \pi, \quad \gamma_{6}=130 \pi\left(\frac{130 \rho_{a}}{2 \mu_{a}}\right)^{1 / 2}, \quad \gamma_{7}=\frac{130 \pi}{4}, \\
& \gamma_{8}=-\left(\frac{0.9 \times 130 \pi}{4}\right)\left(\frac{18 \mu_{a}}{2 \times 130 \rho_{p}}\right)^{1 / 2}, \quad A=3.34 \lambda .
\end{aligned}
$$

According to the definition of $m_{k}, m_{0}$ is the total particle number concentration, and $(\pi / 6) m_{3}$ is the total volume of particles. In the following calculation, we solve the first three moment equations, that is, $k=0,1$, and 2 . The geometric mean particle diameter, $d_{p g}$, and the geometric standard deviation, $\sigma_{p g}$, can be expressed as the function of the first three moments as follows:

$$
d_{p g}=\frac{m_{1}^{2}}{m_{0}^{3 / 2} m_{2}^{1 / 2}}, \quad \sigma_{p g}=\exp \left[\ln \left(\frac{m_{2} m_{0}}{m_{1}^{2}}\right)\right]^{1 / 2} .
$$

\section{Results and Discussions}

3.1. Numerical Specifications. The 4th-order Runge-Kutta method with fixed time step is employed to solve (11) with $k=0,1$, and 2 . The evolutions of PSD are simulated numerically and the effects of RSD on PSD are studied in nine cases. The values of the initialization parameter are listed in Table 1. Case 1 and Case 4 with initial geometric standard deviation $\sigma_{p g 0}=1.5$ are selected to validate the computation codes. Cases 2-5 are selected to obtain the evolution of PSD for clarifying the function by different mechanisms (i.e., Brownian diffusion is dominant for $d_{p g 0}=1-10 \mathrm{~nm}$; both Brownian diffusion and interception are dominant for $d_{p g 0}$ $=0.1-0.5 \mu \mathrm{m}$; and inertial impaction is dominant for $d_{p g 0}=$ 5-8 $\mu \mathrm{m})$. Cases $6-9$ are selected to study the effect of RSD on PSD for a given water content defined by (14) [4], and the corresponding raindrop number concentration, $N_{d}$, is calculated via (15). The computational programs are written 
TABLE 1: The values of the initialization parameter for Cases 1-9.

\begin{tabular}{|c|c|c|c|c|c|c|}
\hline Case & $N_{d} / \mathrm{m}^{-3}$ & $D_{d g} / \mathrm{mm}$ & $\sigma_{d g}$ & $N_{p 0} / \mathrm{m}^{-3}$ & $d_{p g 0} / \mu \mathrm{m}$ & $\sigma_{p g 0}$ \\
\hline 1 & $1.0 \times 10^{5}$ & 0.1 & 1.2 & $1.0 \times 10^{6}$ & 0.001 & $1.2 / 1.5 / 1.8$ \\
\hline 2 & $1.0 \times 10^{5}$ & 0.1 & 1.2 & $1.0 \times 10^{6}$ & 0.01 & $1.2 / 1.5 / 1.8$ \\
\hline 3 & $1.0 \times 10^{5}$ & 0.1 & 1.2 & $1.0 \times 10^{6}$ & 0.1 & $1.2 / 1.5 / 1.8$ \\
\hline 4 & $1.0 \times 10^{5}$ & 0.1 & 1.2 & $1.0 \times 10^{6}$ & 5.0 & $1.2 / 1.5 / 1.8$ \\
\hline 5 & $1.031 \times 10^{7}$ & 0.2 & 1.1 & $1.0 \times 10^{6}$ & $0.01 / 0.5 / 8.0$ & 1.3 \\
\hline 6 & $9.569 \times 10^{4}$ & 0.2 & 1.5 & $1.0 \times 10^{6}$ & $0.01 / 0.5 / 8.0$ & 1.3 \\
\hline 7 & $6.601 \times 10^{5}$ & 0.5 & 1.1 & $1.0 \times 10^{6}$ & $0.01 / 0.5 / 8.0$ & 1.3 \\
\hline 8 & $6.124 \times 10^{3}$ & 0.5 & 1.5 & $1.0 \times 10^{6}$ & $0.01 / 0.5 / 8.0$ & 1.3 \\
\hline 9 & $6.786 \times 10^{4}$ & 0.5 & 1.5 & $1.0 \times 10^{6}$ & $0.01 / 0.5 / 8.0$ & 1.3 \\
\hline
\end{tabular}

using the $\mathrm{C}$ Programming Language and are performed on Microsoft Visual C++ 6.0 complier. Consider

$$
\begin{aligned}
& w=\frac{\pi}{6} \int_{D_{d}} \rho_{w} D_{d}^{3} n\left(D_{d}\right) d D_{d}, \\
& N_{d}=\frac{6 w}{\pi \rho_{w} D_{d g}^{3} \exp \left(9 \sigma_{d g}^{2} / 2\right)} .
\end{aligned}
$$

3.2. Validation of Computation Codes. Particles with diameter $d_{p g 0}=1 \mathrm{~nm}$ and $0.5 \mu \mathrm{m}$ are selected to validate the computation codes. Figure 1 shows the numerical results of particle number concentration based on different collision efficiencies proposed by Slinn [9] and Jung and Lee [29], respectively. The collision efficiency proposed by Slinn is given with a semiempirical formula based on the NavierStokes equation using dimensionless analysis combined with experimental data. While the collision efficiency proposed by Jung and Lee, as shown in (16), is valid for $d_{p g 0}<1 \mu \mathrm{m}$,

$$
E\left(d_{p}, D_{d}\right)=2\left(\frac{\sqrt{3} \pi}{4 P e}\right)^{2 / 3}\left[\frac{(1-\alpha)(3 \sigma+4)}{J+\sigma K}\right]
$$

3.3. Evolution of Particle Geometric Mean Diameter and Geometric Standard Deviation. Figures 2 and 3 show the evolution of particle geometric mean diameter, $d_{p g}$, and geometric standard deviation, $\sigma_{p g}$, in the rainfall process, respectively. From Figure 2 we can see that $d_{p g}$ increases when the initial geometric mean diameter $d_{p g 0}$ is equal to $1 \mathrm{~nm}, 10 \mathrm{~nm}$, and $0.1 \mu \mathrm{m}$, and it decreases when $d_{p g 0}$ is equal to $5 \mu \mathrm{m}$. Thus, we can deduce that there exists one kind of particle diameter, for which $d_{p g}$ never changes in the whole scavenging process. For particles with $d_{p g 0}=1 \mathrm{~nm}$ and $10 \mathrm{~nm}$, $d_{p g}$ grows much faster when the value of $\sigma_{p g 0}$ is larger. And for particles with $d_{p g 0}=5 \mu \mathrm{m}, d_{p g}$ decreases much more rapidly when $\sigma_{p g 0}$ is larger. The region that particle diameter locates between $0.01 \mu \mathrm{m}$ and $2 \mu \mathrm{m}$ is called "Greenfield gap" [30] because the collision efficiency of particles in this region and raindrops is relatively low. While for particles in the "Greenfield gap" (e.g., $\left.d_{p g 0}=0.1 \mu \mathrm{m}\right), d_{p g}$ hardly changes in the whole process. The evolution of $d_{p g}$ in the present study is qualitatively consistent with the result given by Jung et al. [13].

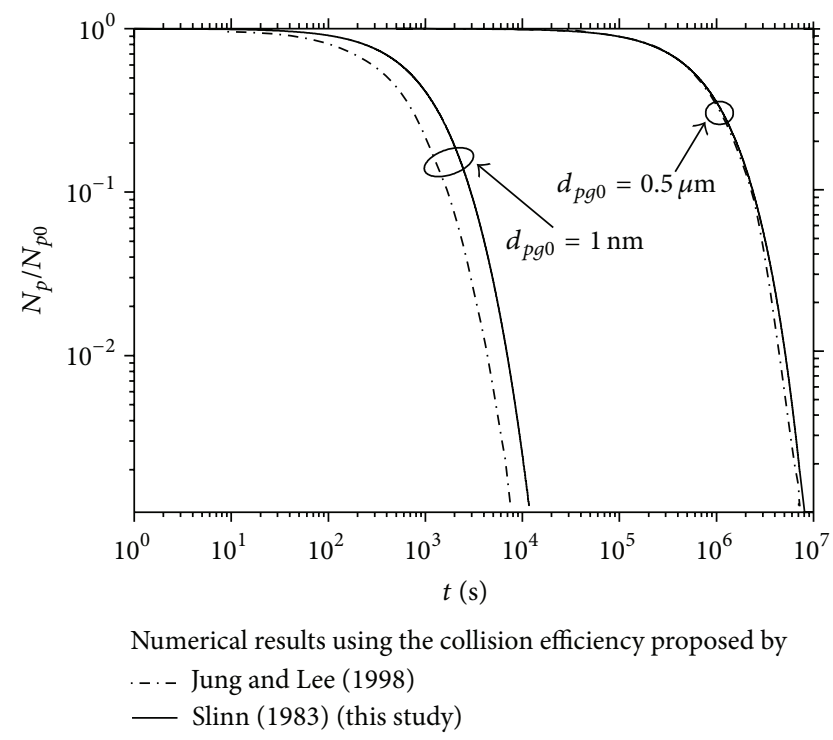

FIGURE 1: Comparison of particle number concentrations based on different collision efficiencies.

In Figure 3, the geometric standard deviation of particle diameter, $\sigma_{p g}$, converges to 1.0 as time goes by in the rainfall process for all particle sizes, which means that the particle size distribution tends to be monodisperse. For particles with the same value of $d_{p g 0}, \sigma_{p g}$ decays much faster when the value of $\sigma_{p g 0}$ is larger. For particles with the same initial value of $\sigma_{p g 0}, \sigma_{p g}$ decreases at an early stage for small particles dominated by the Brownian diffusion, and at a later stage for large particles controlled by the inertial impaction. $\sigma_{p g}$ hardly changes for particles in the "Greenfield gap".

\subsection{Effect of Raindrop Size Distribution on Particle Size} Distribution. The effect of raindrop size distribution (RSD) on the particle size distribution (PSD) is studied using the values of initialization parameter for Cases 6-9 with the given water content $\left(10 \mathrm{~g} / \mathrm{m}^{3}\right)$. Figure 4 shows the evolution of particle number concentration. For Cases 6 and 8 the geometric standard deviation of raindrop diameter, $\sigma_{d g}$, is the same but the geometric mean raindrop diameter, $D_{d g}$, is different. The particle number concentration decreases faster in Case 6 than in Case 8 as shown in Figure 4. For particles 


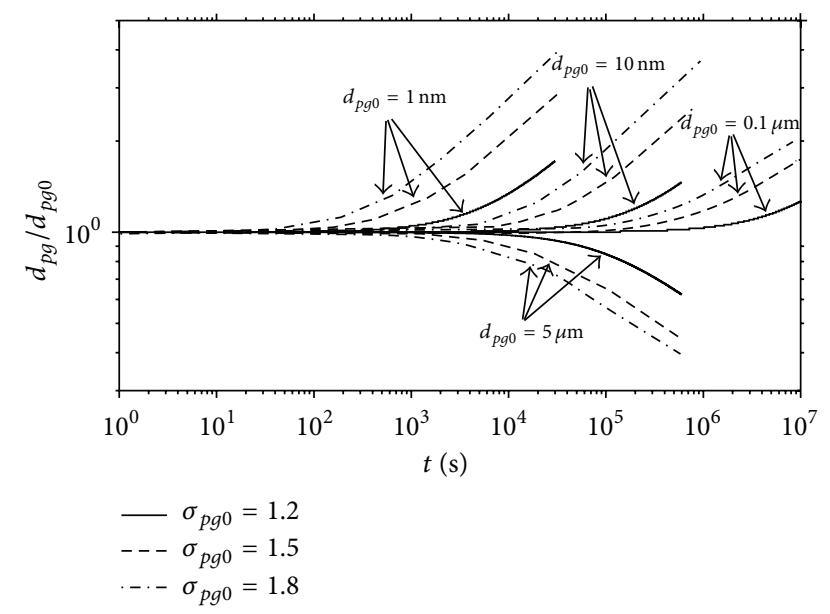

Figure 2: Evolution of particle geometric mean diameter as time progresses for Cases 2-5.

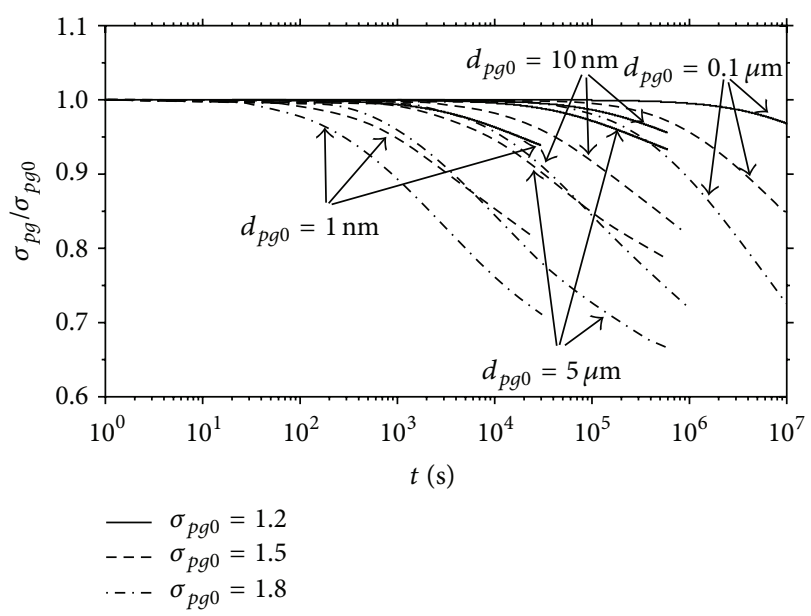

Figure 3: Evolution of geometric standard deviation of particle diameter for Cases 2-5.

with $d_{p g 0}=0.5 \mu \mathrm{m}$ and $d_{p g 0}=8 \mu \mathrm{m}$ in Cases 6 and 8 , the same tendency can be observed, which demonstrates that the RSD in Case 6 can scavenge particles with a much higher efficiency than that in Case 8.

For Cases 8 and 9, the geometric mean raindrop diameter, $D_{d q}$, is the same but the geometric standard deviation of raindrop diameter, $\sigma_{d g}$, is different. It can be seen that the RSD in Case 8 can remove particles with a much higher efficiency than that in Case 9 for any kind of particles. Meanwhile, it takes a much longer time to scavenge particles when $d_{p g 0}=0.5 \mu \mathrm{m}$ in Case 9 than that for any other kind of particle diameters in any case.

Figure 5 shows the effect of RSD on $d_{p g}$ and $\sigma_{p g}$ for particles with $d_{p g 0}=10 \mathrm{~nm}$. It can be seen that $d_{p g}$ increases and $\sigma_{p g}$ decreases in all cases, which is consistent with the results given in Figures 2 and 3. It takes the longest time for $d_{p g} / d_{p g 0}$ and $\sigma_{p g} / \sigma_{p g 0}$ to get the same values in Case 9 , and the shortest time in Case 6. The overall tendency of the effect of RSD on $d_{p g}$ and $\sigma_{p g}$ for particles with $d_{p g 0}=0.5 \mu \mathrm{m}$ and

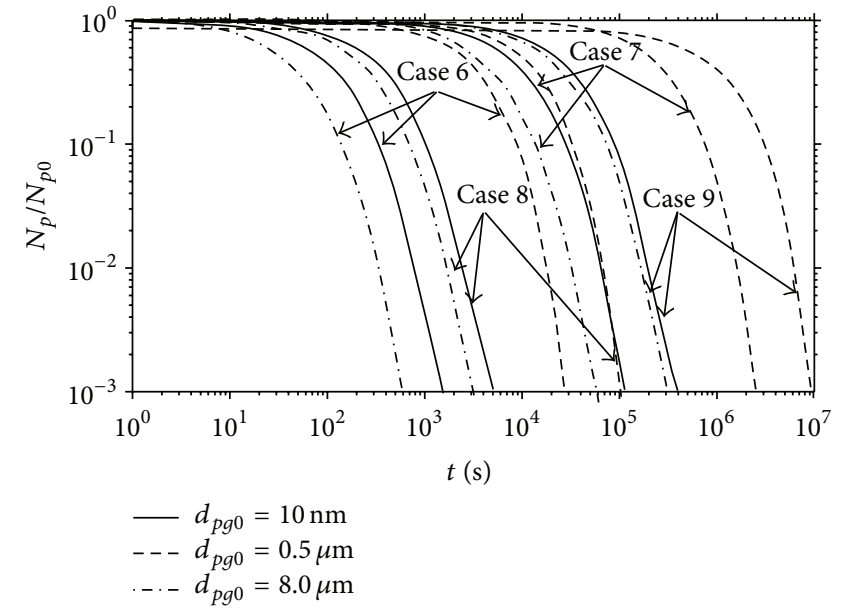

FIgURE 4: Effect of RSD on particle number concentration for Cases 6-9.

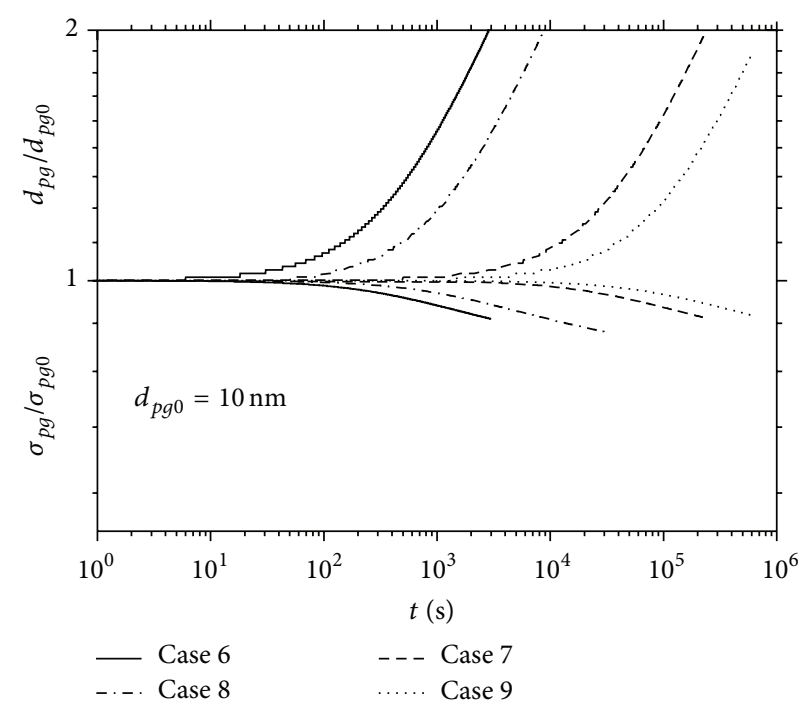

FIgURE 5: Effect of RSD on particle geometric mean diameter and geometric standard deviation $\left(d_{p g 0}=10 \mathrm{~nm}\right)$.

$d_{p g 0}=8 \mu \mathrm{m}$, which is not shown in Figure 5 , is the same as that for particles with $d_{p g 0}=10 \mathrm{~nm}$.

From the above numerical results and analysis, we can see that, for the same water content, the RSD with small $D_{d g}$ can remove the particles more easily than with large $D_{d g}$. The RSD with small $\sigma_{d g}$, that is, a narrow distribution of particle diameter, can collect particles more easily than with large $\sigma_{d g}$. Thus, the RSD in Case 6 is the most efficient in scavenging particles, the RSD in Case 8 takes second place, the RSD in Case 7 the third, and the RSD in Case 9 is the worst, which is consistent with the experimental result [31]. This can be explained in the following way. The raindrop number concentration, $N_{d}$, increases as $D_{d g}$ and $\sigma_{d g}$ decrease with a given water content according to (15), leading to the increase of the total surface area of raindrops, which enhances the probability of capturing small particles dominated by the Brownian diffusion. The intermediate size 
particles controlled by the combined effect of Brownian diffusion and interception depend on the value of $d_{p} / D_{d}$. For the particles with smaller $D_{d g}$ and $\sigma_{d g}, d_{p} / D_{d}$ is larger; that is, the RSD is more monodisperse, which makes the collision efficiency become large as shown in the second term on the right-hand side of (5). Thus, the RSD with smaller $D_{d g}$ and $\sigma_{d g}$ can scavenge intermediate size particles with a much higher efficiency. While for particles with a relatively large size, the scavenging coefficient becomes smaller with larger $D_{d g}$ and $\sigma_{d g}$.

\section{Conclusions}

The removal of aerosol particles in the rainfall process is studied with the periodic moment method. Both the aerosol particle size distribution and raindrop size distribution are assumed to be log-normal. The effects on the collision efficiency of the mechanisms of Brownian diffusion, interception, and inertial impaction have been investigated. The binomial formulas are reexpanded to get a more accurate expression of scavenging coefficient. The first three moments of particle size distribution are simulated and the effects of raindrop size distribution on particle size distribution are studied in nine cases.

The results show that the particle number concentration decreases as time goes by. Particles dominated by Brownian diffusion are removed more easily. The particles in the "Greenfield gap" are the most difficult to be removed. The particle number concentration decreases much more rapidly when particle geometric mean diameter is smaller. In the scavenging process, the particle geometric mean diameter increases when $d_{p g 0}<10 \mathrm{~nm}$, and decreases when $d_{p g 0} \geq$ $1 \mu \mathrm{m}$, but changes a little when $10 \mathrm{~nm}<d_{p g 0}<1 \mu \mathrm{m}$. The geometric standard deviation of particle diameter converges to 1.0 as time progresses for any kind of particles, which means that the particle size distribution tends to be monodisperse, and it decays much faster for particles with large $\sigma_{p g 0}$.

For the same water content, the raindrop size distribution with small $D_{d g}$ can remove particles with a much higher efficiency than that with large $D_{d g}$, and the raindrop size distribution with small $\sigma_{d g}$ can collect particles more easily than with large $\sigma_{d g}$. Particles in the "Greenfield gap" are relatively difficult to scavenge, and a new method is needed to remove it from the air.

\section{Conflict of Interests}

The authors declare that there is no conflict of interests regarding the publication of this paper.

\section{Acknowledgment}

This work was supported by the Major Program of the National Natural Science Foundation of China (11132008).

\section{References}

[1] P. Penttinen, K. L. Timonen, P. Tiittanen, A. Mirme, J. Ruuskanen, and J. Pekkanen, "Number concentration and size of particles in urban air: effects on spirometric lung function in adult asthmatic subjects," Environmental Health Perspectives, vol. 109, no. 4, pp. 319-323, 2001.

[2] J. H. Seinfeld and S. N. Pandis, Atmospheric Chemistry and Physics, Wiley, New York, NY, USA, 1998.

[3] C. H. Jung, Y. P. Kim, and K. W. Lee, "A moment model for simulating raindrop scavenging of aerosols," Journal of Aerosol Science, vol. 34, no. 9, pp. 1217-1233, 2003.

[4] M. Mircea and S. Stefan, "A theoretical study of the microphysical parameterization of the scavenging coefficient as a function of precipitation type and rate," Atmospheric Environment, vol. 32, no. 17, pp. 2931-2938, 1998.

[5] D. M. Chate, P. S. P. Rao, M. S. Naik, G. A. Momin, P. D. Safai, and K. Ali, "Scavenging of aerosols and their chemical species by rain," Atmospheric Environment, vol. 37, no. 18, pp. 2477-2484, 2003.

[6] D. M. Chate and A. K. Kamra, "Collection efficiencies of large water drops collecting aerosol particles of various densities," Atmospheric Environment, vol. 31, no. 11, pp. 1631-1635, 1997.

[7] H. M. Davenport and L. K. Peters, "Field studies of atmospheric particulate concentration changes during precipitation," Atmospheric Environment, vol. 12, no. 5, pp. 997-1008, 1978.

[8] M. Mircea, S. Stefan, and S. Fuzzi, "Precipitation scavenging coefficient: influence of measured aerosol and raindrop size distributions," Atmospheric Environment, vol. 34, no. 29-30, pp. 5169-5174, 2000.

[9] W. G. N. Slinn, "Precipitation scavenging," in Atmospheric Sciences and Power Production, Division of Biomedical Environmental Research, US Department of Energy, Washington, DC, USA, 1983.

[10] C. Andronache, "Estimated variability of below-cloud aerosol removal by rainfall for observed aerosol size distributions," Atmospheric Chemistry and Physics, vol. 3, no. 1, pp. 131-143, 2003.

[11] C. Andronache, "Diffusion and electric charge contributions to below-cloud wet removal of atmospheric ultra-fine aerosol particles," Journal of Aerosol Science, vol. 35, no. 12, pp. 14671482, 2004.

[12] C. Andronache, T. Grönholm, L. Laakso, Y. Phillips, and A. Venäläinen, "Scavenging of ultrafine particles by rainfall at a boreal site: observations and model estimations," Atmospheric Chemistry and Physics, vol. 6, no. 12, pp. 4739-4754, 2006.

[13] C. H. Jung, Y. P. Kim, and K. W. Lee, "Analytic solution for polydispersed aerosol dynamics by a wet removal process," Journal of Aerosol Science, vol. 33, no. 5, pp. 753-767, 2002.

[14] S. Y. Bae, C. H. Jung, and Y. Pyo Kim, "Development and evaluation of an expression for polydisperse particle scavenging coefficient for the below-cloud scavenging as a function of rain intensity using the moment method," Journal of Aerosol Science, vol. 37, no. 11, pp. 1507-1519, 2006.

[15] D. L. Marchisio, R. D. Vigil, and R. O. Fox, "Implementation of the quadrature method of moments in CFD codes for aggregation-breakage problems," Chemical Engineering Science, vol. 58, no. 15, pp. 3337-3351, 2003.

[16] R. E. Sampson and G. S. Springer, "Condensation on and evaporation from droplets by a moment method," Journal of Fluid Mechanics, vol. 36, no. 3, pp. 577-584, 1969. 
[17] M. Yu, J. Lin, and T. Chan, "A new moment method for solving the coagulation equation for particles in Brownian motion," Aerosol Science and Technology, vol. 42, no. 9, pp. 705-713, 2008.

[18] M. Yu and J. Lin, "Taylor-expansion moment method for agglomerate coagulation due to Brownian motion in the entire size regime," Journal of Aerosol Science, vol. 40, no. 6, pp. 549562, 2009.

[19] S. H. Park and K. W. Lee, "Analytical solution to change in size distribution of polydisperse particles in closed chamber due to diffusion and sedimentation," Atmospheric Environment, vol. 36, no. 35, pp. 5459-5467, 2002.

[20] L. Jianzhong, S. Xing, and Y. Zhenjiang, "Effects of the aspect ratio on the sedimentation of a fiber in Newtonian fluids," Journal of Aerosol Science, vol. 34, no. 7, pp. 909-921, 2003.

[21] J. Lin, P. Lin, and H. Chen, "Research on the transport and deposition of nanoparticles in a rotating curved pipe," Physics of Fluids, vol. 21, no. 12, Article ID 122001, 11 pages, 2009.

[22] M. Yu, J. Lin, and T. Chan, "Numerical simulation of nanoparticle synthesis in diffusion flame reactor," Powder Technology, vol. 181, no. 1, pp. 9-20, 2008.

[23] M. Z. Yu, J. Z. Lin, and T. L. Chan, "Effect of precursor loading on non-spherical $\mathrm{TiO} 2$ nanoparticle synthesis in a diffusion flame reactor," Chemical Engineering Science, vol. 63, no. 9, pp. 2317-2329, 2008.

[24] S. E. Pratsinis, "Simultaneous nucleation, condensation, and coagulation in aerosol reactors," Journal of Colloid and Interface Science, vol. 124, no. 2, pp. 416-427, 1988.

[25] K. W. Lee and B. Y. H. Liu, "Theoretical study of aerosol filtration by fibrous filters," Aerosol Science and Technology, vol. 1, no. 2, pp. 147-161, 1982.

[26] G. Feingold and Z. Levin, "The lognormal fit to raindrop spectra from frontal convective clouds in Israel," Journal of Climate and Applied Meteorology, vol. 25, no. 10, pp. 1346-1363, 1986.

[27] E. R. Whitby and P. H. McMurry, "Modal aerosol dynamics modeling," Aerosol Science and Technology, vol. 27, no. 6, pp. 673-688, 1997.

[28] S. Y. Bae, C. H. Jung, and Y. P. Kim, "Derivation and verification of an aerosol dynamics expression for the below-cloud scavenging process using the moment method," Journal of Aerosol Science, vol. 41, no. 3, pp. 266-280, 2010.

[29] C. H. Jung and K. W. Lee, "Filtration of fine particles by multiple liquid droplet and gas bubble systems," Aerosol Science and Technology, vol. 29, no. 5, pp. 389-401, 1998.

[30] S. Greenfield, "Rain scavenging of radioactive particulate matter from the atmosphere," Journal of Meteorology, vol. 14, no. 2, pp. 115-125, 1957.

[31] K. W. Lai, N. Dayan, and M. Kerker, "Scavenging of aerosol particles by a falling water drop," Journal of Atmospheric Science, vol. 35 , no. 4 , pp. $674-682,1978$. 


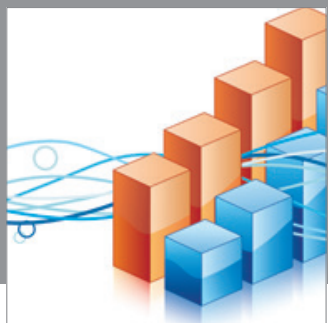

Advances in

Operations Research

mansans

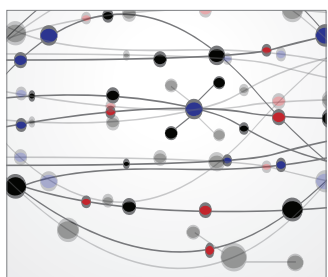

The Scientific World Journal
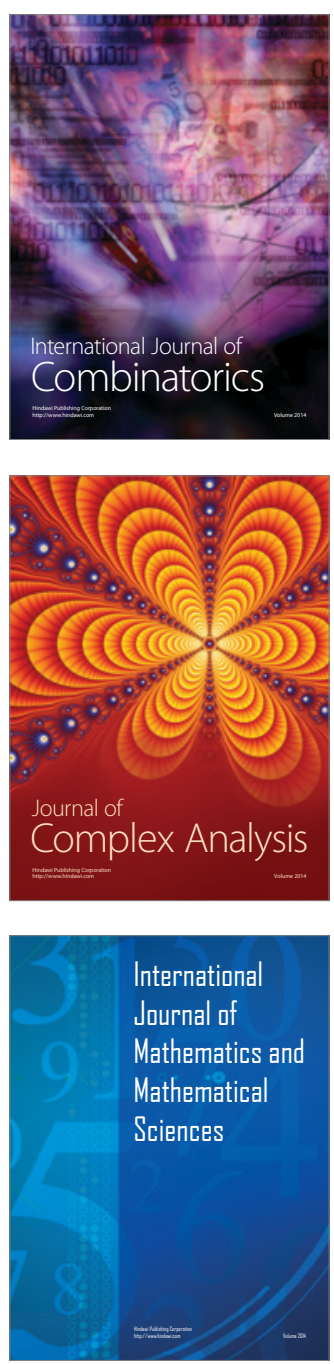
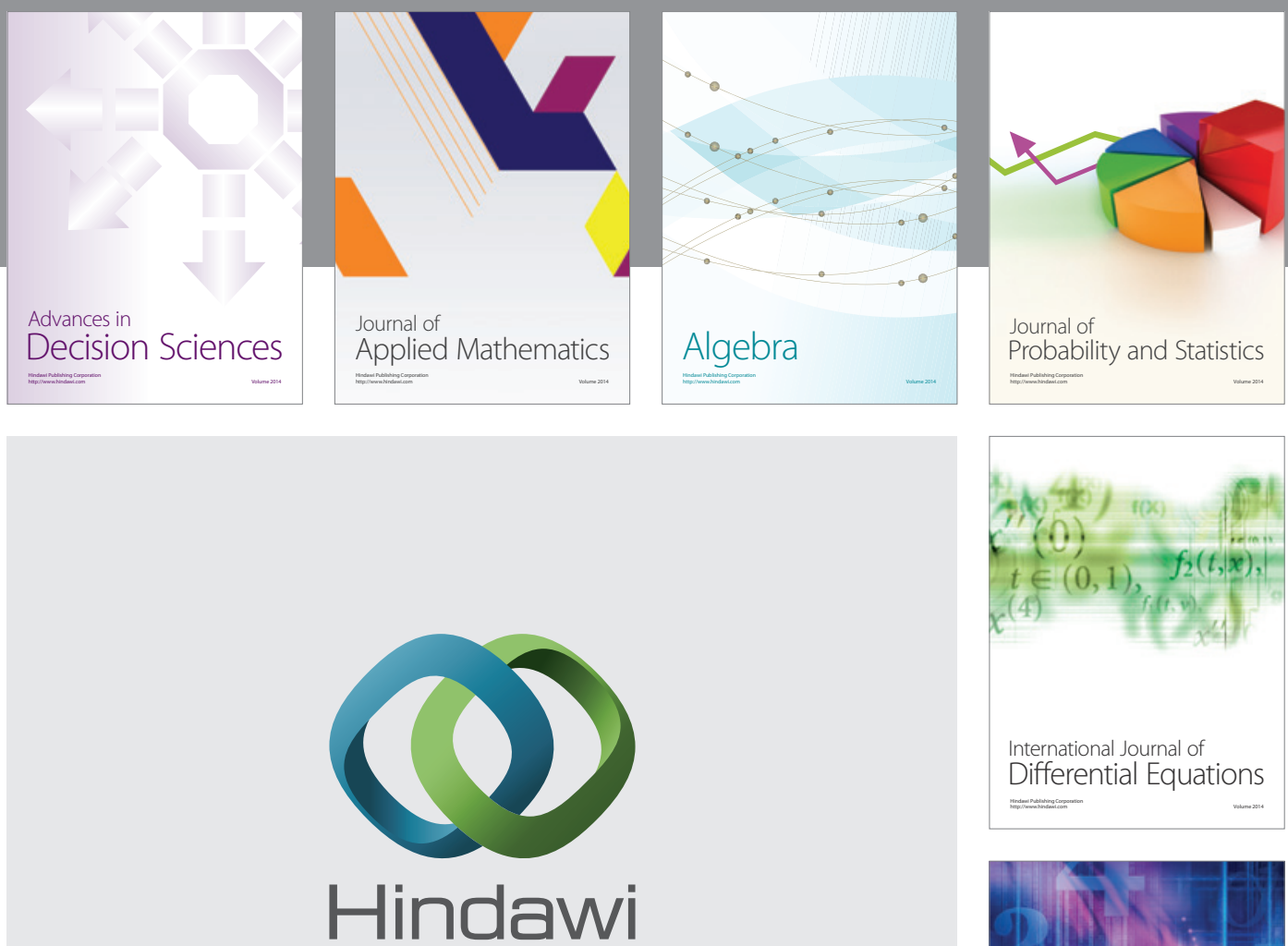

Submit your manuscripts at http://www.hindawi.com
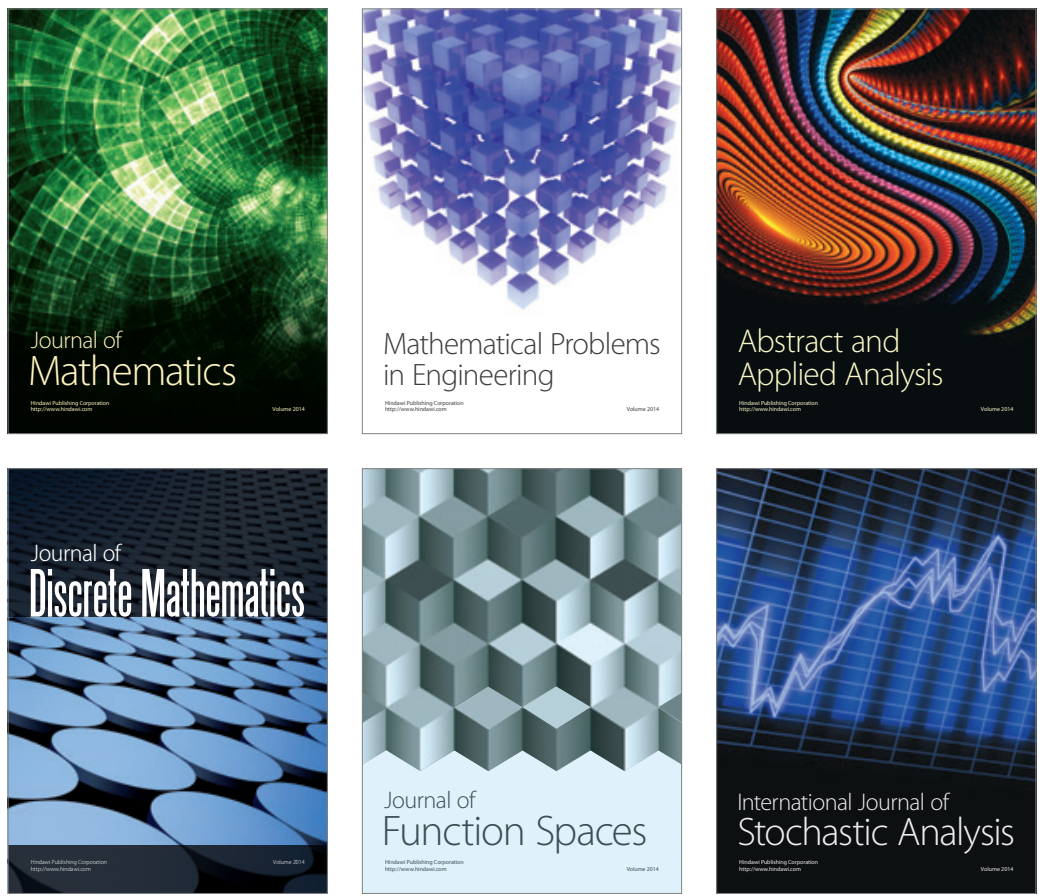

Journal of

Function Spaces

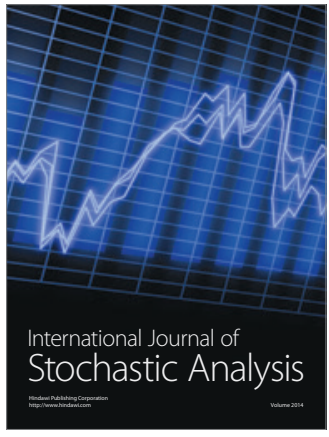

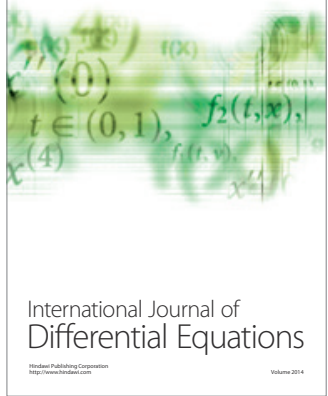
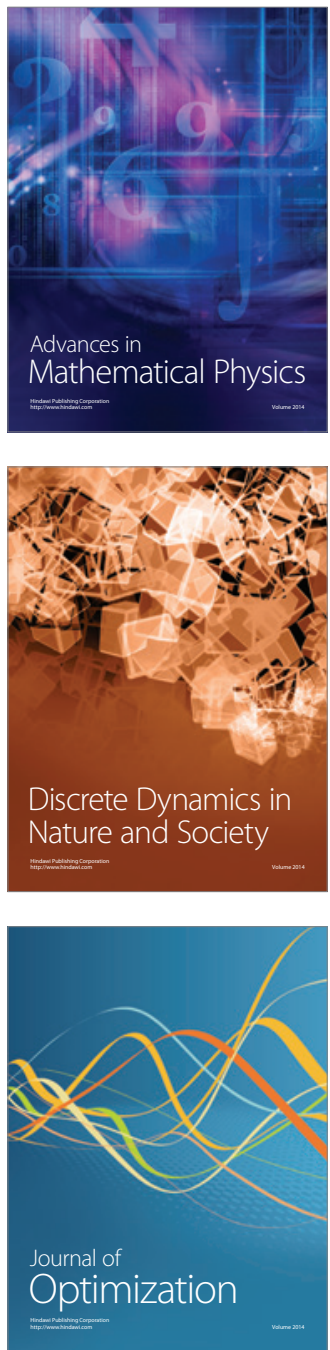